\title{
First-Principle and Experimental Study of a Gadolinium-Praseodymium-Cobalt Pseudobinary Intermetallic Compound
}

\author{
Jon Goldsby, ${ }^{1}$ Sai Raj, ${ }^{1}$ Sivaraman Guruswamy, ${ }^{2}$ and Daniel David Azbill ${ }^{2}$ \\ ${ }^{1}$ Materials and Structures Division, NASA Glenn Research Center, Cleveland, OH 44135, USA \\ ${ }^{2}$ Department of Metallurgical Engineering, University of Utah, Salt Lake City, UT 84112, USA \\ Correspondence should be addressed to Jon Goldsby; jon.c.goldsby@nasa.gov
}

Received 23 February 2015; Revised 27 May 2015; Accepted 28 May 2015

Academic Editor: Iwan Kityk

Copyright (C) 2015 Jon Goldsby et al. This is an open access article distributed under the Creative Commons Attribution License, which permits unrestricted use, distribution, and reproduction in any medium, provided the original work is properly cited.

First-principles methods were used to determine the magnetic state of a simulated cobalt-based binary alloy $(\mathrm{Gd}, \mathrm{Pr}) \mathrm{Co}_{17}$ along with its corresponding lattice parameters and density. The resulting composition was fabricated using two methods arc-melting and induction-melting and compared with the calculated values. The induction-melted samples showed greater homogeneity and successfully produced the $\mathrm{R}_{2} \mathrm{Co}_{17}$ structure. Calculated values qualitatively predict ferromagnetic behavior and lattice parameters to be within a low percent. The development of magnetic alloys with the assistance of computational methods promises faster development of new functional materials.

\section{Introduction}

Permanent magnets are unique in their ability to convert energy, whether mechanical to mechanical energy or electric to mechanical energy. They, in themselves, have no moving parts, no coolant, nor lubrication and hence, inherently a higher reliability. Permanent magnets can also be utilized to create electrical power from gas turbine engines, with their relatively high electromotive force contributing to reducing the overall system volume $[1,2]$. Recent studies have shown that a significant reduction in fuel burn for future aircraft can be achieved by developing hybrid-electric airplanes. One factor that limits the power density of the permanent magnet electric motors is the temperature capability of the magnets. As the motor temperature increases during operation, current magnets lose their magnetic properties. The best high-temperature magnetic material available today is $\mathrm{Sm}_{2}(\mathrm{Co}, \mathrm{Fe})_{17}$, which has a temperature limit of $400^{\circ} \mathrm{C}$ for long-duration operation. Currently, magnets are developed with trial and error and empirical methods. These commercial magnets are developed with design criteria specific to their application, as an example for automotive or audio service. However, for aviation, the requirements are more stringent and permanent magnets must perform in extreme environments. Computational methods can be used to find potential high-performing systems and to filter out alloy compounds, which show little promise for meeting the requirements. In addition, the computational methods also give very good predictions of the materials' properties such as the magnetic moments, their saturation, physical density, and stiffness, which are relevant to further fabrication. A high coercivity is desirable for long duration and strength. Furthermore, coercivity decreases with an increase in temperature. Therefore, decreasing the coercivity sensitivity to temperature can result in an increase in a permanent magnet's use temperature. The motivation for this investigation is to develop a high-temperature, permanent magnetic material with a high Curie temperature and with a capability for use above $500^{\circ} \mathrm{C}$. Instead of maximizing the Curie temperature [3], this approach will attempt to reduce the temperature coefficient of the magnetization [4] by selecting atoms $\mathrm{R}$ in the $\mathrm{R}_{2} \mathrm{Co}_{17}$ system that have net magnetic moments in opposite to the relative net spin of the cobalt in the crystal structure. The cobalt-based alloy was chosen because of their inherently high Curie temperatures $[5,6]$. 


\section{Methods}

2.1. Computational Methods. The selection of proper alloy composition has to this point been somewhat speculative in nature based upon empirical experimentation. With the use of computational methods, material properties can be evaluated and optimized before the material is processed allowing an optimized structure to be realized quickly. This saves enormous time and efforts of experimentally assessing properties of multiple changes in composition and structure. The density functional theory has been proved to be one of the accurate methods of calculating the electronic properties of the solid [7-15]. The approach consists of physics-based computational simulations using the Cambridge Serial Total Energy Package (CASTEP) [16] to identify candidate materials based on lattice parameters and their electronic configuration as possible high-temperature magnets. We used a computing platform consisting of a Hewlett-Packard Z820 workstation that is sufficient to carry out the computations of this investigation. The workstation consists of 32 core processors of 64-bit operating system and $192 \mathrm{~GB}$ of random access memory. In this study Accelrys Materials Studio version 6 was used as the client interface. Starting with the base structure, a geometric optimization was performed to determine the initial lattice parameters and density. In this calculation, to obtain accurate band structures, the generalized gradient approximation (GGA) [17] was used along with ultrasoft pseudopotentials to represent the atoms. Specifically, for this geometric optimization, the Perdew-Burke-Ernzerhof potential for solids (PBEsol) was used [18, 19]. The cut-off energy was $470 \mathrm{eV}$ with a fine K-point $4 \times 4 \times 4$ mesh. The calculations were also carried out using a projector augmented wave (PAW) [20] method using Materials Design Inc. MedeA incorporating the Vienna Ab Initio Simulation Package (VASP 5.2) [21] as the computational engine. The calculations were based on density functional theory and the GGA-PBE exchangecorrelation functional. In this case a spin-orbit magnetic calculation using "accurate" precision and an increased plane wave cut-off energy of 348.35 . The actual k-spacing was $0.338 \times 0.338 \times 0.338$ per angstrom which corresponds to a $3 \times 3 \times 3$ mesh.

2.2. Alloy Fabrication Methods. The alloy was fabricated by two methods, electric-induction and arc-melting of the metal alloy components, followed by powder metallurgy processing and testing the high-temperature magnetic capability. The appropriate compositions will be arc-melted to form the basic ingot. This will be followed by powder metallurgy processing involving crushing, milling, powder compaction, and sintering. The samples were then thermally treated. Microstructure was examined with the goal of increased magnetic and mechanical durability in extreme environments. Once a composition is selected a pilot charge, consisting of 10 to 20 grams, was fabricated. High-purity (99.5\%) metals (Alfa Aesar, Ward Hill, MA) of praseodymium, gadolinium, and cobalt were consolidated in an electric arc furnace. A tri-arc-melting furnace manufactured by Materials Research Furnace (Suncook, $\mathrm{NH}$ ) was used to melt small buttons. Because the charge was

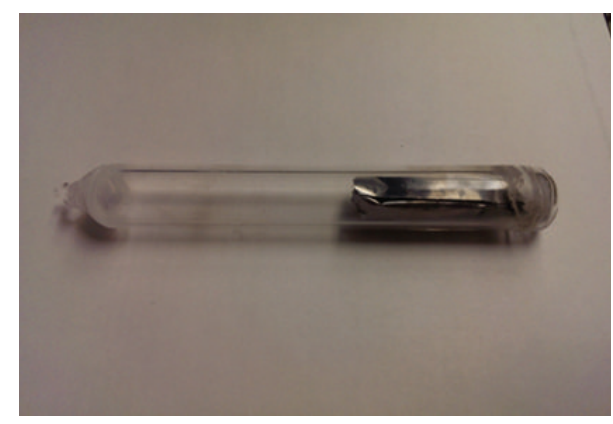

FIGURE 1: Sealed quartz tube containing cold and pressed alloy pellet ready for sintering.

placed on a water-cooled copper hearth, minimal contamination was anticipated. Tungsten electrodes powered by a heavy-duty cycle welding power source delivered 60 to 100 amperes of current to melt the sample. To aid in mixing, the samples are flipped several times and remelted. The entire melting sequence is carried out under flowing argon gas to prevent oxidation. The samples are subsequently homogenized at various temperatures and times from 2 to 100 hours at two-thirds of the melting temperature of the constituent materials. The annealed ingot sample was then milled wet in a protective kerosene fluid using a SPEX mill using stainless steel container with $6 \mathrm{~mm}$ stainless steel balls as milling media. Milling durations were between 30 and 75 minutes. After milling, the kerosene was decanted. The powder was then washed in acetone and methanol and stored in a sealed container. Pellets $(6 \mathrm{~mm}$ diameter $\times 6 \mathrm{~mm}$ height $)$ were formed using a polyethylene glycol bind. Alloy powders were dissolved initially in acetone as a lubricant/binder (11.5 wt.\%) and then cold-pressed with $1500 \mathrm{~kg} / \mathrm{cm}^{2}$ of pressure. The sample was heated under argon gas to $325^{\circ} \mathrm{C}$ to remove the binder. Green body pellets were then sealed under argon in a quartz vial using an oxyacetylene torch as seen in Figure 1 and sintered $1190^{\circ} \mathrm{C}$ for several hours. A mechanical pump is used to evacuate a glass tube (sealed at one end and connected to the gas/vacuum line at the other) to less than about 0.1 Torr. The tubing is then backfilled with ultra-highpurity argon to +5 psi and evacuated, and the cycle is repeated about 15 times before the sealing is done. The vial length is about 4 inches so that the sample is not heated during sealing. With annealing time, the magnetization observed should increase and saturates at some time duration, typically within 48 hours. Magnetization measurements on as-cast and powder processed samples were performed using a Lakeshore 7307 vibrating sample magnetometer at room and elevated temperatures to evaluate temperature coefficient and coercivity.

\section{Results and Discussion}

3.1. Computational Simulation. For the initial crystal structure space group, $\mathrm{R}-3 \mathrm{~m}$ was selected, which is representative of $\mathrm{R}_{2} \mathrm{Co}_{17}$ compounds. To increase the speed of computations, the equivalent rhombohedra representation with its fewer atoms per cell was used to optimize the geometry and 
TABLE 1: Integrated spin density comparison used to determine magnetic ordering of the simulated crystal unit cell.

\begin{tabular}{llccc}
\hline Parameter & Ferromagnetic & Paramagnetic & Antiferromagnetic & Ferrimagnetic \\
\hline $2 *$ Integrated spin density & Nonzero, the same magnitude & Zero & Zero & Nonzero \\
$2 *$ Integrated |spin density| & Nonzero, the same magnitude & Zero & Nonzero & Nonzero, larger magnitude \\
\hline
\end{tabular}

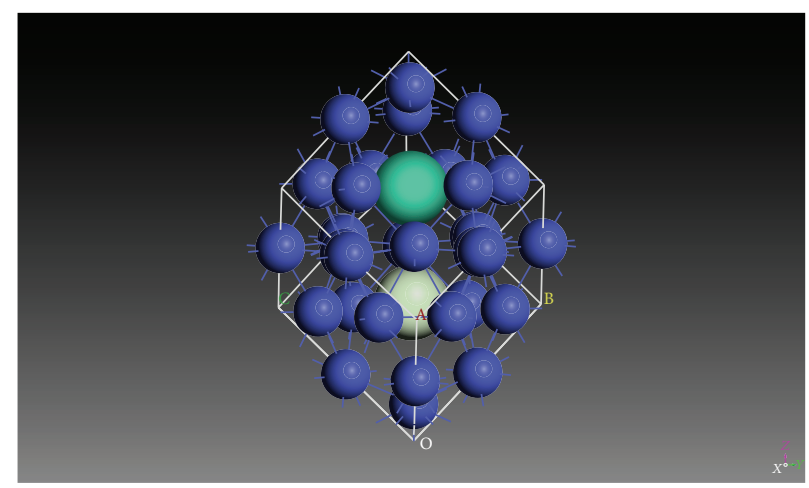

FIGURE 2: Rhombohedral representation of praseodymium (light pale blue), cobalt (blue), and gadolinium (green) system where atoms are rendered as spheres with radii related to van der Waals' radii of the respective atoms.

then reverted back to its conventional hexagonal form to compare lattice parameters with experimental values. The self-convergent field limit was set to $2 \times 10^{-5}$ electron-volts per atom and achieved convergence within 30 iterations with final enthalpy energy of $-22008 \mathrm{eV}$ after 12 hours of computations. The X-ray diffraction Rietveld analysis of fabricated alloy indicated a crystallite structure and size of $\mathrm{R}-3 \mathrm{~m}$ and $16.75 \mathrm{~nm}$, respectively, for the induction-melted sample. The analysis also gives the room temperature lattice parameters as $a=b=8.412$ angstroms (A) and $c=$ 12.229 (A). These numbers are larger, but comparable to the calculated values of $a=b=8.291$ (A) and $c=12.160$ (A). These differences may reflect the fact that density functional theory computations are athermal in nature; hence phenomena like thermal expansion are not taken into account. Nonetheless, these results confirm the correct selection of the initial structure for the computational simulation. Figure 2 denotes the graphical representation of the praseodymium, cobalt, and gadolinium system under study. The relative atomic radii are depicted in this figure where the two lanthanide atoms dominate the unit crystal cell volume. Nearest-neighbor cobalt-cobalt distance is 2.576 angstroms while Figure 3 gives the net spin density of states of the alloys explicitly illustrating the net spin of the electron states where $E_{\mathrm{Fermi}}$ denotes the Fermi level of this material [22]. The participating electron orbitals $\operatorname{Pr} 4 f^{3} 5 s^{2} 5 p^{6} 6 s^{2}, G d 4 f^{7} 5 s^{2} 5 p^{6} 5 d^{1} 6 s^{2}$, and Co $3 d^{7} 4 s^{2}$ are hybridized with the $s, d$, and $f$ orbitals electrons contributing the most to the material's magnetic moment. The charge density is given by the difference between conduction electron populations, which are spin up or spin down, in accordance with Pauli's exclusion principle, and hence a measure of the electron spin unbalance. Spin density is defined as the number of electrons per unit crystal volume; therefore the integral is an electron count, the difference between the numbers of spin-up and spin-down electrons in the crystal cell. The entire sum of the electron populations or integrated spin densities therefore provides a qualitative prediction of the nature of the magnetic ordering within the material. A comparison [23] between this value and its absolute magnitude can be used to determine the magnetic state based upon the criteria given in Table 1. A Bohr magneton is defined as a unit of magnetic dipole moment. However, since every electron has a spin dipole moment of 2.00232 Bohr magnetons, the total magnetic moment expressed in units of Bohr Magneton is numerically equivalent to twice the integral spin density. Hence, for this alloy, the predicted total magnetic dipole moment is 37 Bohr magnetons.

3.2. Magnetic Properties. Magnetization measurement of the gadolinium, praseodymium, and cobalt $(\mathrm{Pr}, \mathrm{Gd}) \mathrm{Co}_{17}$ alloy powders is obtained after annealing the cast ingot and coarsely milling to a powder with an average size of about $30 \mu \mathrm{m}$. The hysteresis curve obtained from this measurement is shown in Figure 4 giving the saturation magnetization and coercivity of the new ferromagnetic compound. The preliminary results of the magnetization reveal a saturation value of 0.9 Tesla or 93 amperes $*$ meter $^{2}$ per $\mathrm{kg}$ under an applied external magnetic field of 9975 Gauss. This is considerably less than the predicted CASTEP value of 158 amperes * meter $^{2} / \mathrm{kg}$ or the 110 amperes $*$ meter $^{2} / \mathrm{kg}$ VASP prediction based upon the calculated total dipole moment. However, the computed values are essentially those of a single crystal, and its predictions do not include possible grain boundary and other polycrystalline phases. While the results of VASP-PAW method give results in slightly better agreement with the experimental values than the CASTEP-ultrasoft potential, both methods are sufficient to gain a qualitative understanding of the candidate magnetic systems. The normalized temperature of the specific magnetization (Figure 5) remains near zero for the alloys tested. Table 3 gives the calculated compositions for the various charge weights and 


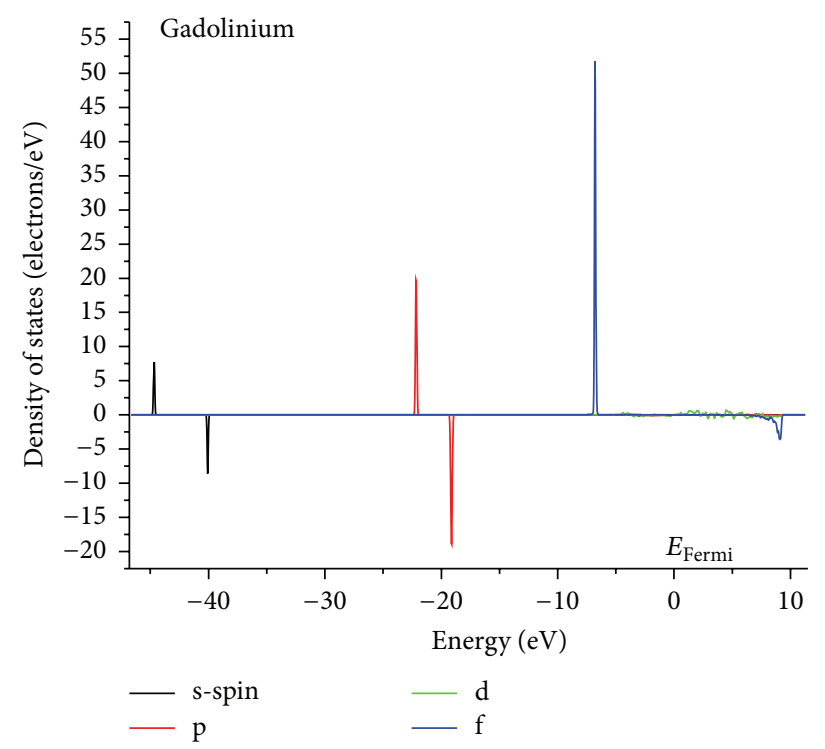

(a)

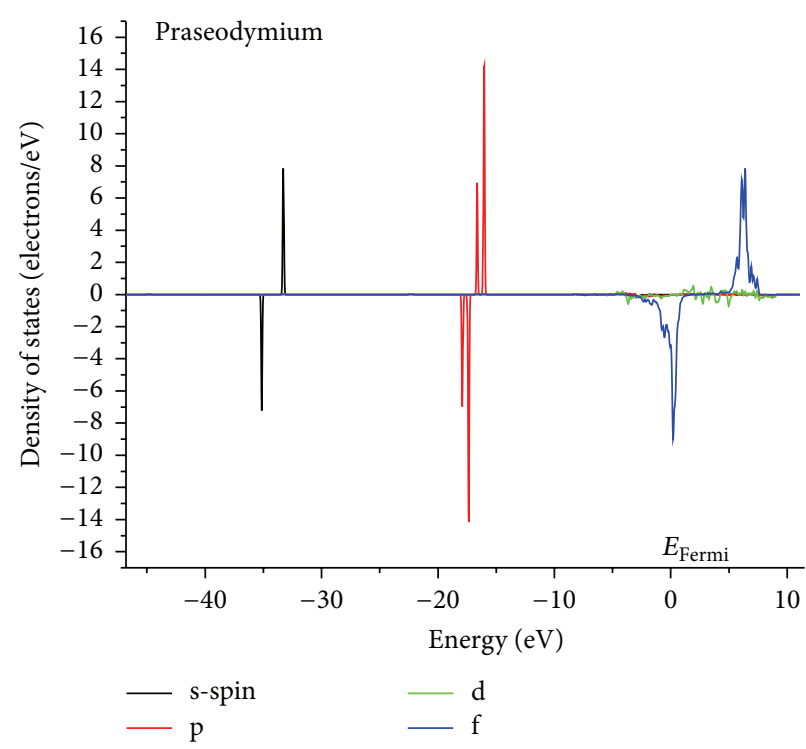

(b)

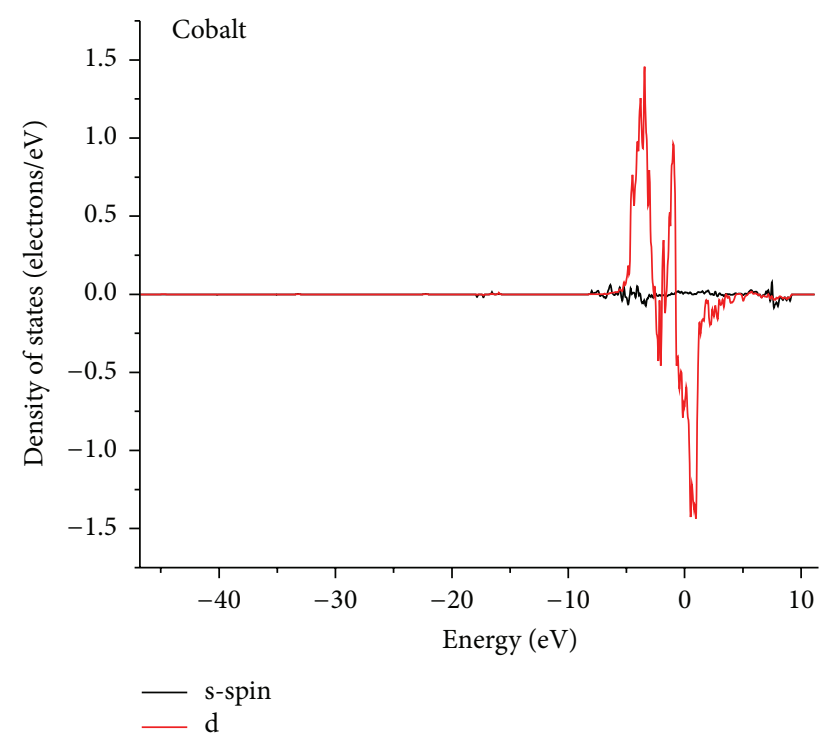

(c)

FIGURE 3: Calculated densities of electron states spin decomposed and symmetry-resolved illustrating the difference between the spin-up (positive) and spin-down (negative) electron states, which give rise to the ferromagnetism of this alloy.

the actual compositions. The results indicate that very little material has been lost due to vaporization from either the electric arc- or induction-melting process. Figure 6 illustrates the mass-specific magnetic moments for the alloys fabricated with the two techniques. The induction-melted material decreases more relative to the arc-melted alloy. This may be attributed to the magnetization behavior in the arc-melted sample being dominated by cobalt not significantly interacting with the lanthanide atoms as would be expected in a stoichiometric $\mathrm{R}_{2} \mathrm{CO}_{17}$ compound.

3.3. Microstructure. The particle size of the powder after several minutes of milling can be seen in micrograph
(Figures 7(a) and 7(b)). The alloys melted from the two methods differ in their fractured particles morphologies. The arcmelted sample (Figure 7(a)) forms a single size particle size distribution and appears granular, whereas the inductionmelted sample (Figure 7(b)) breaks into a more tabularplatelet morphology. While the arc-melted sample average particle size is 10 micrometers, the induction-melted sample appears to have a bimodal distributed particle size between 10 and 40 micrometers. The density of the induction-melted sample was determined using the Archimedes immersion procedure and was found to be $8.46 \mathrm{~g} / \mathrm{cm}^{3}$; this compares favorably with the calculated values (Table 2). Figures 8 and 9 give postprocessed microstructural information of 


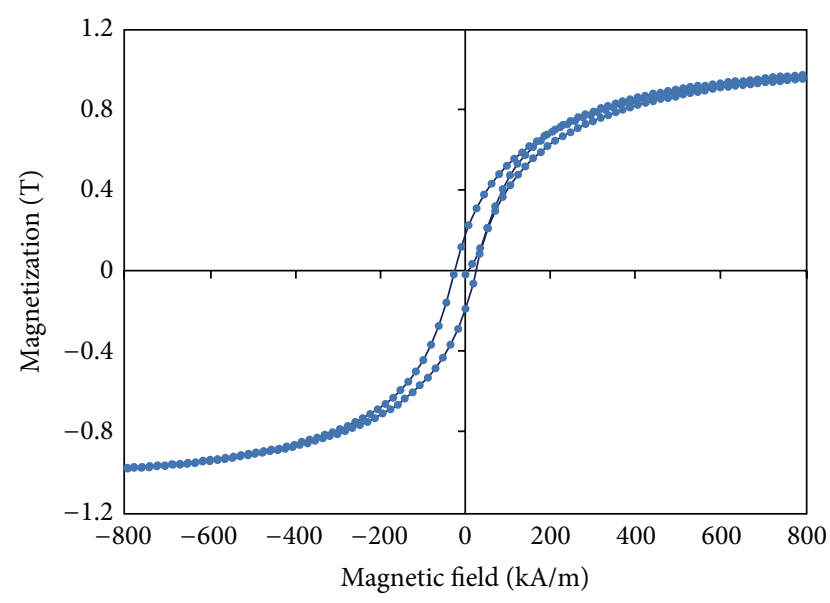

(a)

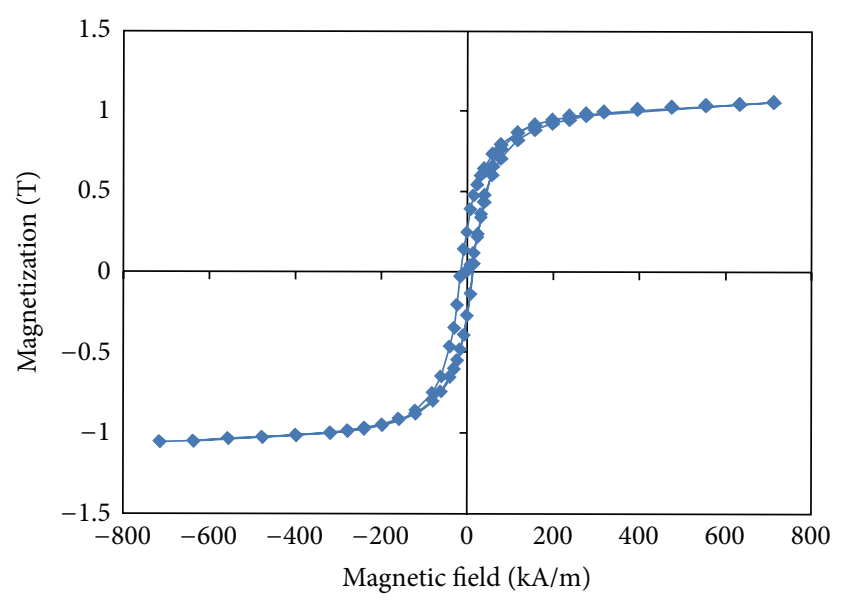

(b)

FIGURE 4: The hysteresis curves for the annealed (a) arc- and (b) induction-melted (Gd,Pr)Co ${ }_{17}$ coarse powders.

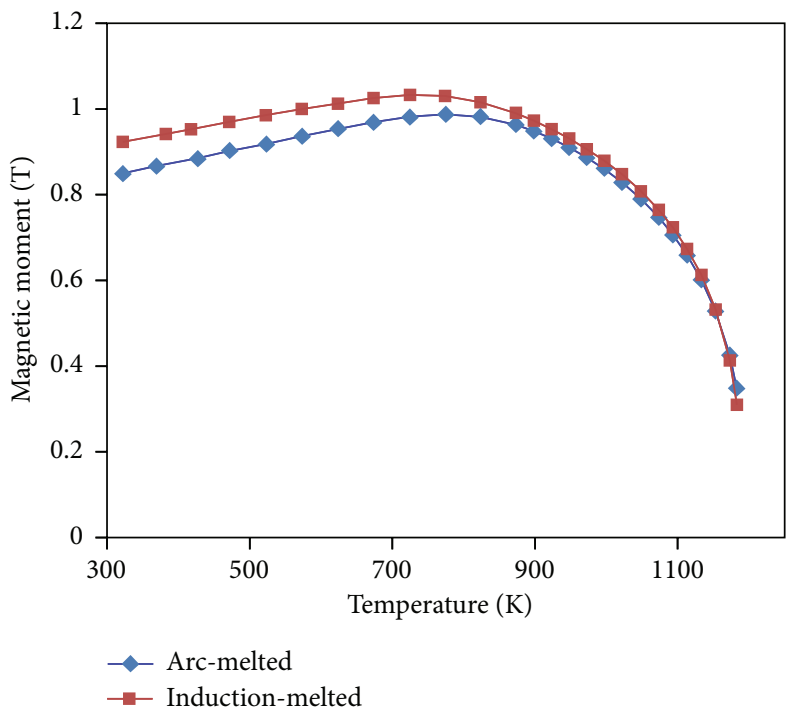

FIGURE 5: Comparison of temperature dependent specific magnetic moments between arc- and induction-melted $(\mathrm{Pr}, \mathrm{Gd}) \mathrm{Co}_{17}$.

the arc-melted $(\mathrm{Pr}, \mathrm{Gd}) \mathrm{Co}_{17}$ alloy. Figure 8 gives an overall view of the material; immediately evident is the relatively high porosity. The final processed and tested density for the sintered materials is only $4.29 \mathrm{~g} / \mathrm{cm}^{3}$, thereby yielding a material with $48 \%$ porosity. Pressure assisted or spark plasma sintering will likely be needed to yield a more fully dense sample. To determine the compositions within the various phases, energy dispersive spectroscopy (EDS) was used. The spectrum reveals concentrated areas of praseodymium and gadolinium within the cobalt matrix (Figure 10). High lanthanide concentrations, such as these, indicate that the electric arc-melting process used in this study, even with a maximum applied current of 400 amperes, was not sufficient to fully melt and homogenize the magnetic alloys to a satisfactory level. In addition, the porosity found in the postsintered material means that full density was not achieved with nonpressure sintering. In contrast Figure 11(a) shows no evidence of lanthanide segregation. The microstructure of the postannealed induction-melted sample appears smooth with only pockets of cobalt, which are likely remnant dendrites from the solidification process and then isolated during the homogenization anneal. The cobalt appears to be raised relative to the surrounding matrix. This is likely an artifact of the nonacid etched polishing process in preparing the sample for metallographic analysis, but it is also an indication that the cobalt is harder than the surrounding matrix. Figure 11(b) illustrates that at higher magnification the cobalt phase contains postannealed twins, while EDS (Figure 12) on sections $\mathrm{A}, \mathrm{D}$, and $\mathrm{E}$ reveals a mixture of lanthanide and cobalt metals and $\mathrm{B}$ and $\mathrm{C}$ are pure cobalt. In addition, $\mathrm{X}$-ray diffraction patterns give the proper $\mathrm{R}_{2} \mathrm{Co}_{17}$ phase; hence the gadolinium praseodymium cobalt pseudobinary intermetallic compound was readily formed using the electric induction-melting method. 
TABle 2: Predicted and experimental material parameters of the $(\mathrm{Gd}, \mathrm{Pr}) \mathrm{Co}_{17}$ ferromagnetic alloy.

\begin{tabular}{lccc}
\hline $\mathrm{GdPrCo}_{17}$ & Density $\left(\mathrm{g} / \mathrm{cm}^{3}\right)$ & Lattice parameter $($ angstrom $)$ & Magnetization $($ amperes $*$ meter \\
\hline CASTEP & 8.37 & $a=b=8.46, c=12.47$ & 158 \\
\hline VASP & 8.82 & $a=b=8.35, c=12.17$ & 110 \\
\hline Experiment & 8.46 & $a=b=8.41, c=12.23$ & 93 \\
\hline
\end{tabular}

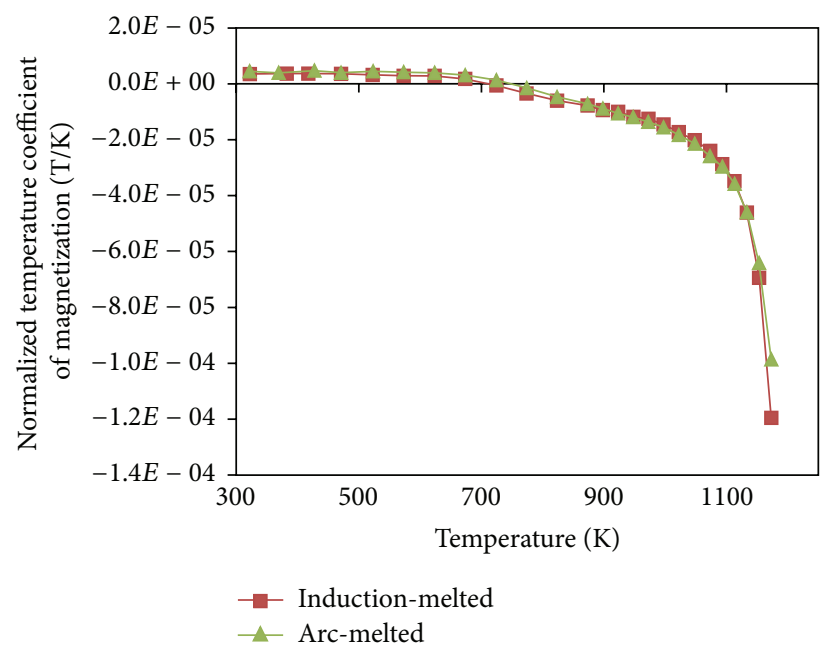

FIGURE 6: Temperature stability of magnetic properties.

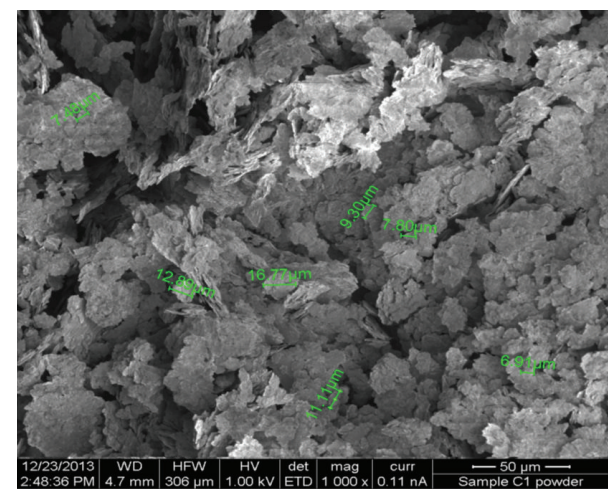

(a)

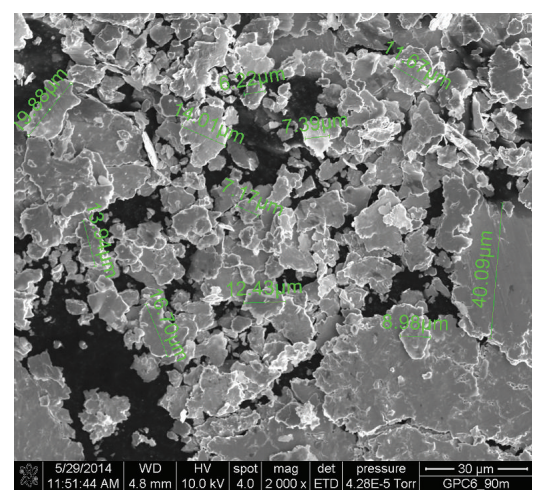

(b)

Figure 7: A micrograph of milled praseodymium gadolinium cobalt alloy powder (a) arc-melted and (b) induction-melted before cold pressing and sintering.

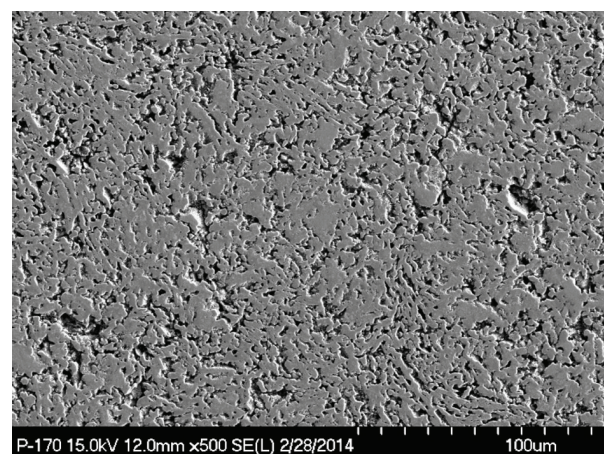

FIGURE 8: Micrograph of pressureless sintered arc-melted powdered $(\mathrm{Pr}, \mathrm{Gd}) \mathrm{Co}_{17}$ showing numerous pores. 


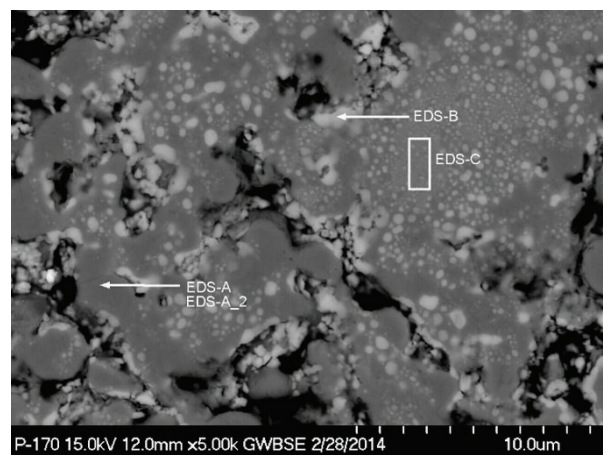

FIGURE 9: Micrograph of $(\mathrm{Pr}, \mathrm{Gd}) \mathrm{Co}_{17}$ showing lanthanide metal concentrations in the arc-melted alloy.
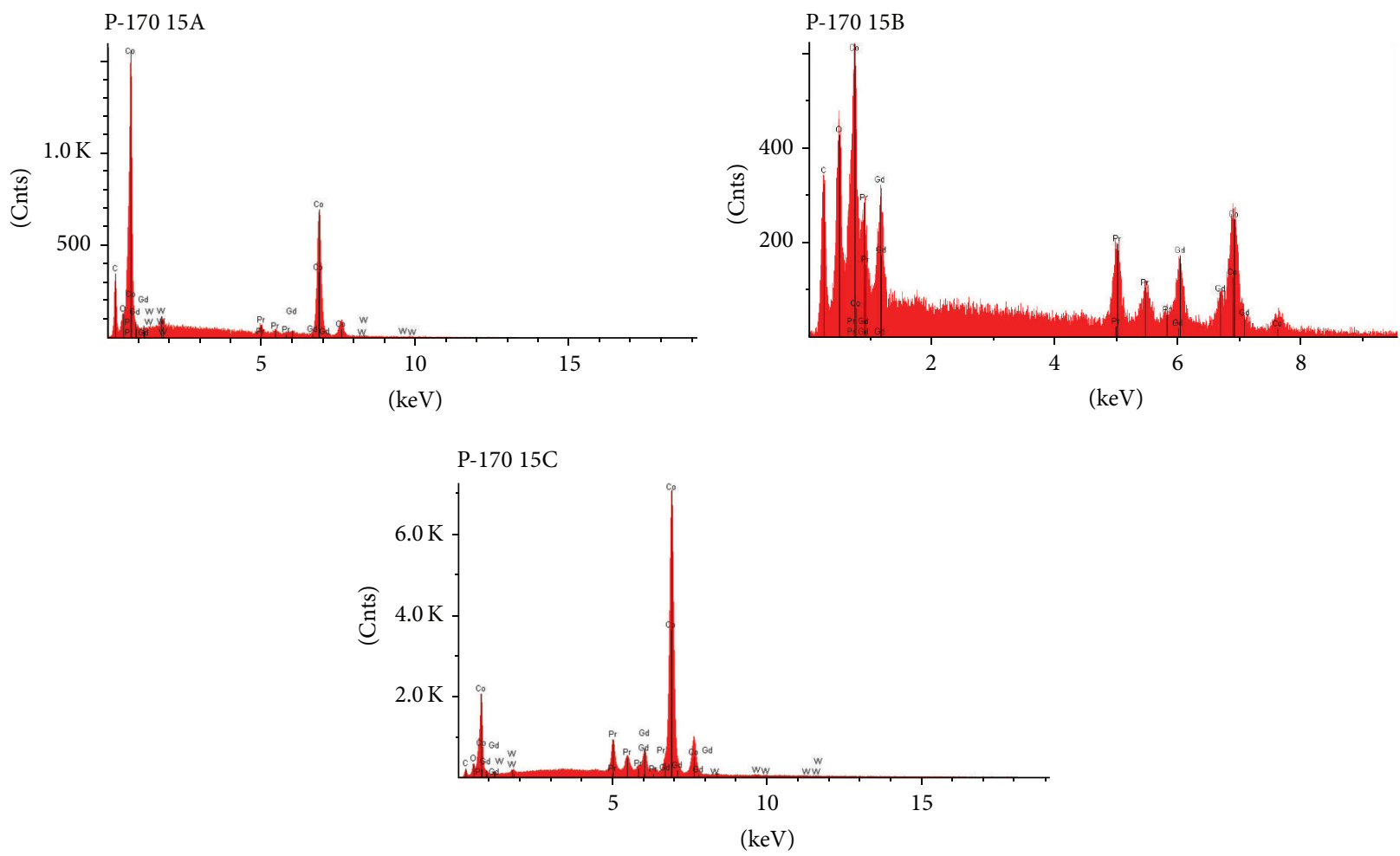

FIGURE 10: EDS spectrum for Figure 9 illustrating lanthanide-rich regions.

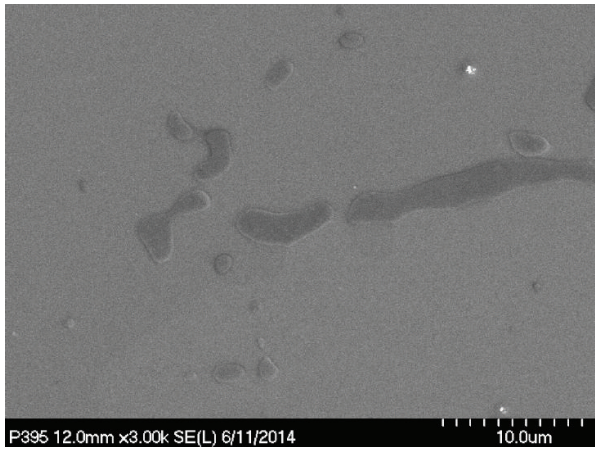

(a)

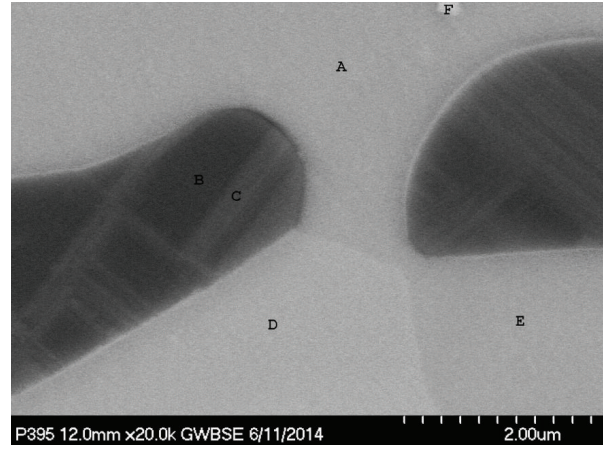

(b)

FIGURE 11: Micrograph (a) of the induction-melted (Gd,Pr) $\mathrm{Co}_{17}$ after annealing (b) and magnified to illustrate cobalt-rich regions. 

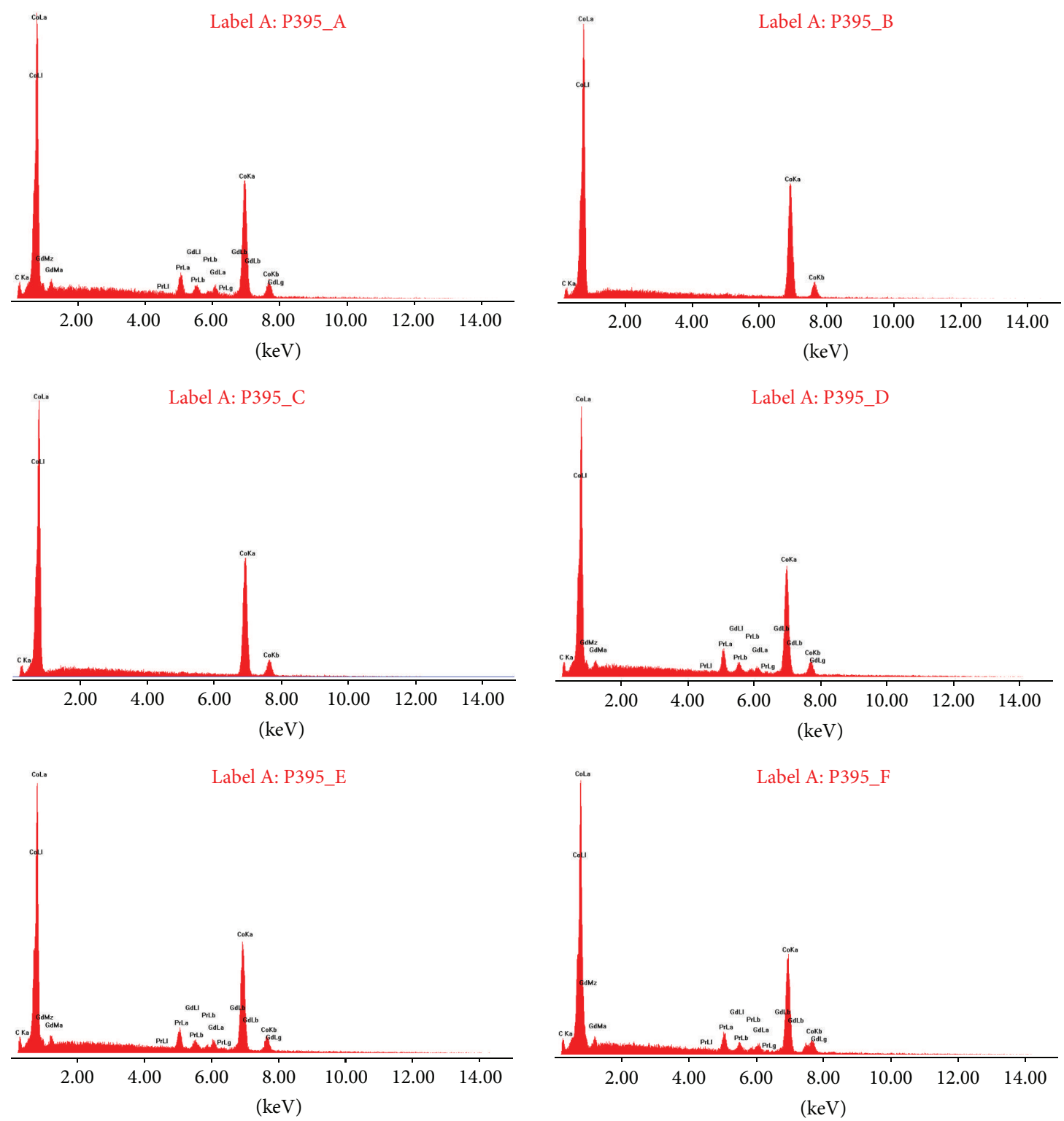

FIGURE 12: EDS spectrum of Figure 11, two-phase region.

TABLE 3: Nominal alloy compositions in wt $\%$ and analytical results in parentheses and stoichiometric compositions in brackets.

\begin{tabular}{lccc}
\hline (Pr,Gd) $\mathrm{Co}_{17}$ & $\mathrm{Pr}$ & $\mathrm{Gd}$ & $\mathrm{Co}$ \\
weight percent & {$[10.8]$} & {$[12.4]$} & {$[76.8]$} \\
\hline \multirow{2}{*}{ Electric arc-melted alloy } & 10.8 & 12.1 & 77.1 \\
& $(11.2)$ & $(12.7)$ & $(76.1)$ \\
\hline \multirow{2}{*}{ Induction-melted alloy } & 11.05 & 12.28 & 76.67 \\
& $(10.9)$ & $(11.9)$ & $(77.2)$ \\
\hline
\end{tabular}

\section{Conclusions}

Density functional theory-based calculations allowed predictions for the properties for a gadolinium-praseodymiumcobalt pseudobinary intermetallic compound. The simulation has successfully predicted the magnetic state of the compound as well as the lattice parameters, density. Induction-melted samples of the alloy result in better solution and phase formation of this $\mathrm{R}_{2} \mathrm{Co}_{17}$ compound compared to arcmelting. Computational methods can aid in the development of new magnetic materials.

\section{Conflict of Interests}

The authors declare that there is no conflict of interests regarding the publication of this paper.

\section{References}

[1] C. Zwyssig and J. W. Kolar, "Design considerations and experimental results of a 100 W, $500000 \mathrm{rpm}$ electrical generator," Journal of Micromechanics and Microengineering, vol. 16, no. 9, pp. S297-S302, 2006. 
[2] W. U. N. Fernando, M. Barnes, and O. Marjanovic, "Direct drive permanent magnet generator fed AC-DC active rectification and control for more-electric aircraft engines," IET Electric Power Applications, vol. 5, no. 1, pp. 14-27, 2011.

[3] R. F. Sabiryanov and S. S. Jaswal, "Ab Initio calculations of the Curie temperature of complex permanent-magnet materials," Physical Review Letters, vol. 79, no. 1, pp. 155-158, 1997.

[4] A. S. Kim, "Design of high temperature permanent magnets," Journal of Applied Physics, vol. 81, no. 8, pp. 5609-5611, 1997.

[5] K. Watanabe, T. Kaneko, and S. Ohnuma, "Temperaturedependence of magnetic-properties in Co-Pt, Fe-Pt and Cr-Pt permanent-magnet alloy system," Journal of the Japan Institute of Metals, vol. 56, no. 12, pp. 1495-1500, 1992.

[6] J. Zhou, R. Skomski, C. Chen, G. C. Hadjipanayis, and D. J. Sellmyer, "Sm-Co-Cu-Ti high-temperature permanent magnets," Applied Physics Letters, vol. 77, no. 10, pp. 1514-1516, 2000.

[7] A. H. Reshak, I. V. Kityk, and S. Auluck, "Energy band structure and density of states for $\mathrm{BaBiBO}_{4}$ nonlinear optical crystal," Journal of Alloys and Compounds, vol. 460, no. 1-2, pp. 99-102, 2008.

[8] Y. Saeed, S. Nazir, A. H. Reshak, and A. Shaukatd, "Firstprinciples study of spin-polarized electronic band structures in ferromagnetic $\mathrm{Zn}_{1-x} \mathrm{TM}_{x} \mathrm{~S}(\mathrm{TM}=\mathrm{Fe}$, Co and Ni)," Journal of Alloys and Compounds, vol. 508, no. 2, pp. 245-250, 2010.

[9] A. H. Reshak, H. Kamarudin, and S. Auluck, "Bismuthcontaining semiconductors: linear and nonlinear optical susceptibilities of $\mathrm{GaAs}_{1-x} \mathrm{Bi}_{x}$ alloys," Journal of Alloys and Compounds, vol. 509, no. 40, pp. 9685-9691, 2011.

[10] H. S. Saini, M. Singh, A. H. Reshak, and M. K. Kashyap, "Emergence of half metallicity in Cr-doped GaP dilute magnetic semiconductor compound within solubility limit," Journal of Alloys and Compounds, vol. 536, pp. 214-218, 2012.

[11] A. H. Reshak, H. Kamarudin, and S. Auluck, "Dispersion of the linear and nonlinear optical susceptibilities of disilver germanium sulfide from DFT calculations," Journal of Materials Science, vol. 48, no. 5, pp. 1955-1965, 2013.

[12] A. H. Reshak, I. V. Kityk, O. V. Parasyuk et al., "X-ray photoelectron spectrum, X-ray diffraction data, and electronic structure of chalcogenide quaternary sulfide $\mathrm{Ag}_{2} \mathrm{In}_{2} \mathrm{GeS}_{6}$ : experiment and theory," Journal of Materials Science, vol. 48, no. 3, pp. 13421350, 2013.

[13] A. H. Reshak, I. V. Kityk, O. V. Parasyuk, H. Kamarudin, and $\mathrm{S}$. Auluck, "Influence of replacing $\mathrm{Si}$ by $\mathrm{Ge}$ in the chalcogenide quaternary sulfides $\mathrm{Ag}_{2} \mathrm{In}_{2} \mathrm{Si}(\mathrm{Ge}) \mathrm{S}_{6}$ on the chemical bonding, linear and nonlinear optical susceptibilities, and hyperpolarizability," The Journal of Physical Chemistry B, vol. 117, no. 8, pp. 2545-2553, 2013.

[14] A. H. Reshak, H. Huang, H. Kamarudin, and S. Auluck, "Alkali-metal/alkaline-earth-metal fluorine beryllium borate $\mathrm{NaSr}_{3} \mathrm{Be}_{3} \mathrm{~B}_{3} \mathrm{O}_{9} \mathrm{~F}_{4}$ with large nonlinear optical properties in the deep-ultraviolet region," Journal of Applied Physics, vol. 117, no. 8, Article ID 085703, 2015.

[15] A. H. Reshak, "Specific features of electronic structures and optical susceptibilities of molybdenum oxide," RSC Advances, vol. 5, no. 28, pp. 22044-22052, 2015.

[16] M. D. Segall, P. J. D. Lindan, M. J. Probert et al., "First-principles simulation: ideas, illustrations and the CASTEP code," Journal of Physics: Condensed Matter, vol. 14, no. 11, pp. 2717-2744, 2002.

[17] J. P. Perdew, K. Burke, and M. Ernzerhof, "Generalized gradient approximation made simple," Physical Review Letters, vol. 77, no. 18 , pp. 3865-3868, 1996.
[18] J. P. Perdew, A. Ruzsinszky, G. I. Csonka et al., "Restoring the density-gradient expansion for exchange in solids and surfaces," Physical Review Letters, vol. 100, no. 13, Article ID 136406, 2008.

[19] J. Paier, M. Marsman, and G. Kresse, "Why does the B3LYP hybrid functional fail for metals?" Journal of Chemical Physics, vol. 127, no. 2, Article ID 024103, 2007.

[20] G. Kresse and D. Joubert, "From ultrasoft pseudopotentials to the projector augmented-wave method," Physical Review B, vol. 59 , no. 3, pp. $1758-1775,1999$.

[21] G. Kresse and J. Furthmüller, "Efficient iterative schemes for ab initio total-energy calculations using a plane-wave basis set," Physical Review B, vol. 54, no. 16, pp. 11169-11186, 1996.

[22] J. Robertson, K. Xiong, and S. J. Clark, "Band structure of functional oxides by screened exchange and the weighted density approximation," Physica Status Solidi B: Basic Solid State Physics, vol. 243, no. 9, pp. 2054-2070, 2006.

[23] R. F. L. Evans, W. J. Fan, P. Chureemart, T. A. Ostler, M. O. A. Ellis, and R. W. Chantrell, "Atomistic spin model simulations of magnetic nanomaterials," Journal of Physics: Condensed Matter, vol. 26, no. 10, Article ID 103202, 2014. 

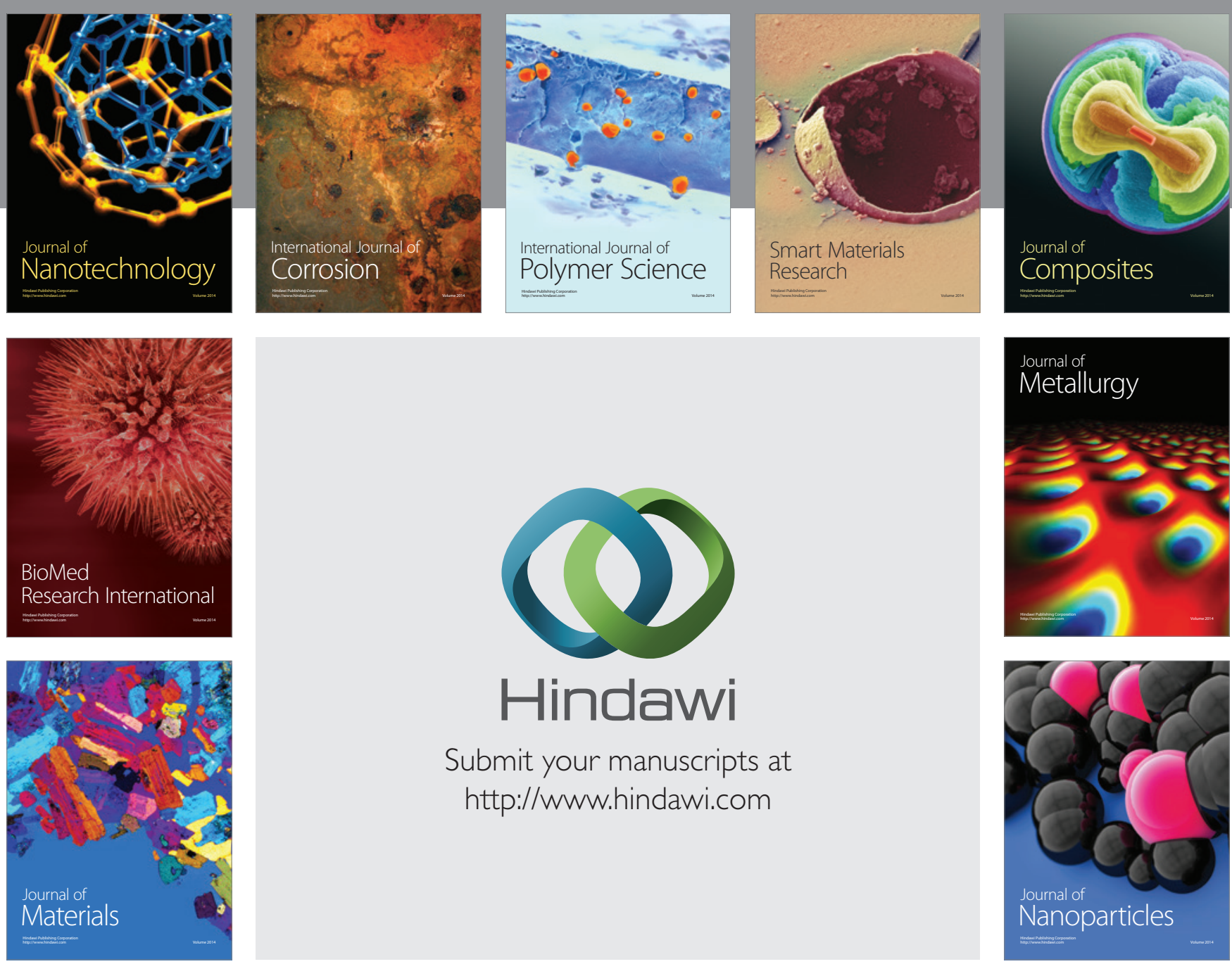

Submit your manuscripts at http://www.hindawi.com
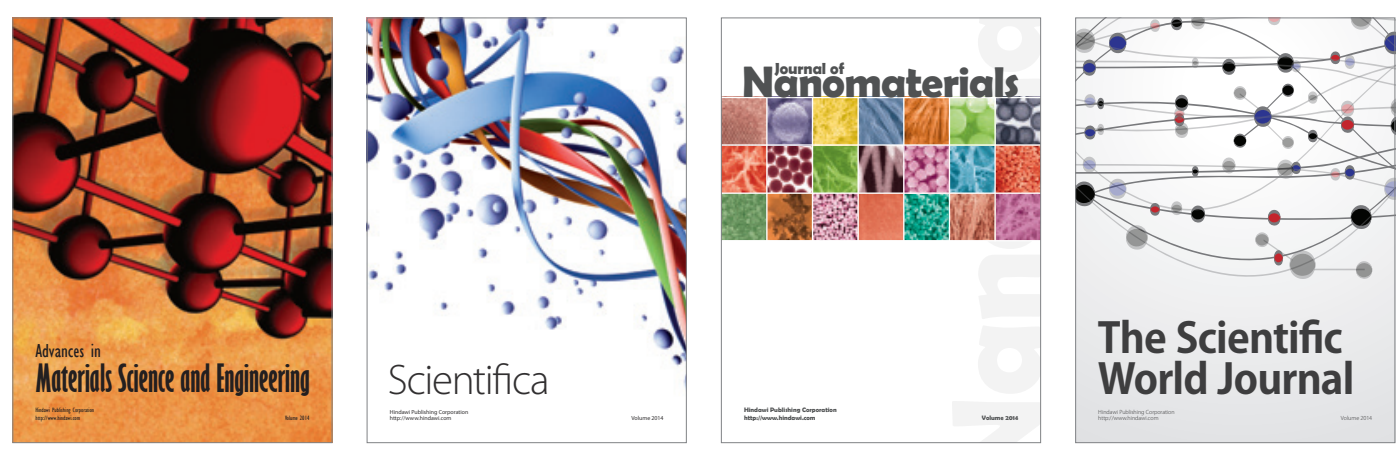

\section{The Scientific World Journal}
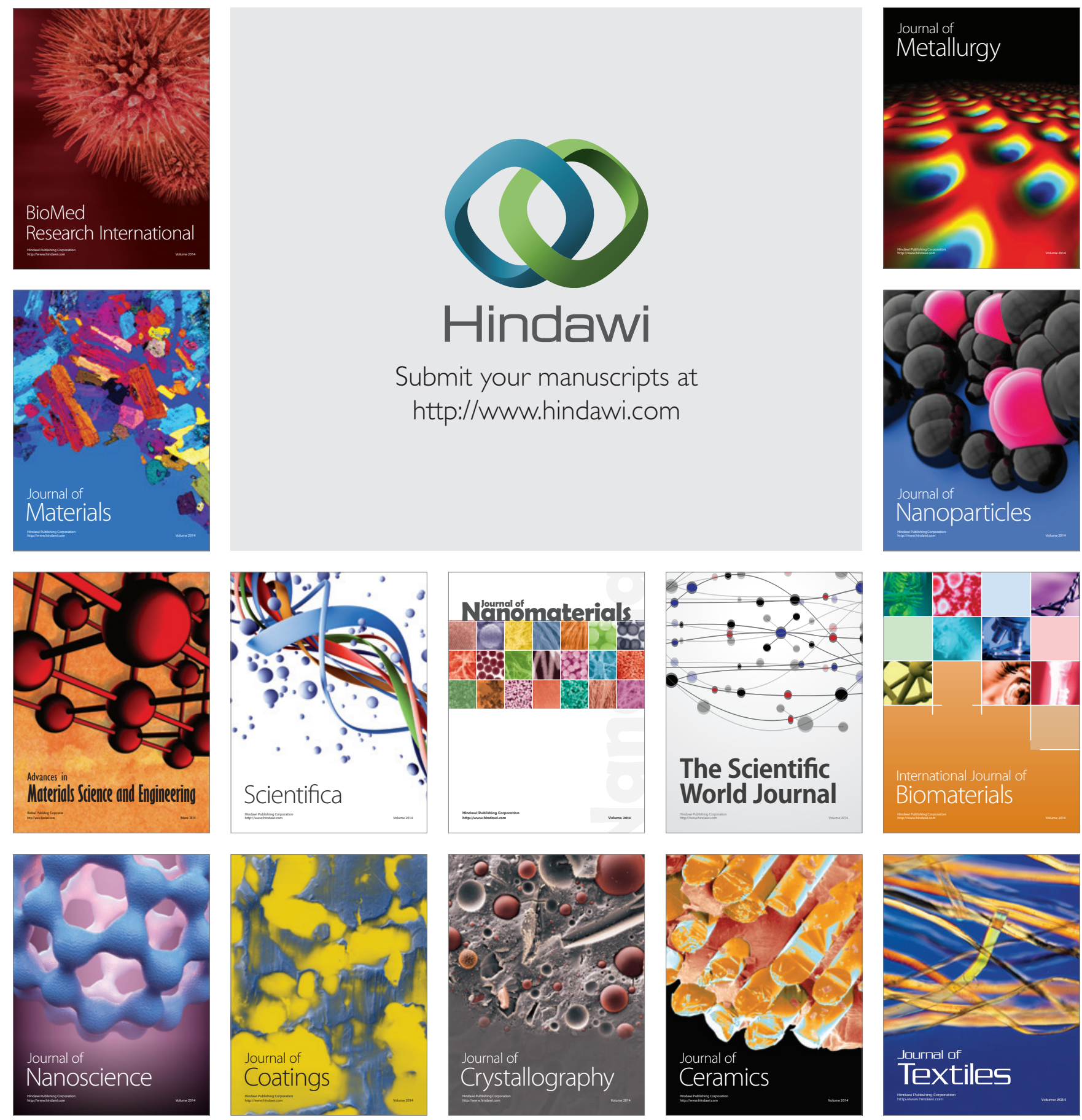